Eur. J. Stat. 2 (2022) 4

doi: 10.28924/ada/stat.2.4

\title{
Some Resultats on Optimal Allocation of Policy Limits and Deductibles: Mixture Model
}

\author{
Bouhadjar Meriem ${ }^{1}$, Halim Zeghdoudi ${ }^{1, *}{ }^{\oplus}$, Abdelali Ezzebsa $^{2}$ \\ ${ }^{1}$ LaPS Laboratory, Badji-Mokhtar University, BP12, Annaba 23000-Algeria \\ bouhadjarmeriem.math@yahoo.com, halimzeghdoudi77@gmail.com \\ ${ }^{2}$ Department of Mathematics, University May 8,1945-Guelma, Algeria \\ aezzebsa@gmail.com \\ *Correspondence: halimzeghdoudi77@gmail.com
}

\begin{abstract}
The main purpose of this paper is to introduce and investigate stochastic orders of scalar products of random vectors. We study the problem of finding maximal expected utility for some functional on insurance portfolios involving some additional (independent) randomization. Furthermore, applications in policy limits and deductible are obtained, we consider the scalar product of two random vectors which separates the severity effect and the frequency effect in the study of the optimal allocation of policy limits and deductibles. In that respect, we obtain the ordering of the optimal allocation of policy limits and deductibles when the dependence structure of the losses is unknown. Our application is a further study of $[1-6]$.
\end{abstract}

\section{INTRODUCTION}

\section{Introduction and motivation}

In recent years modern actuarial theory and risk theory have attracted attention and are widely studied in various fields. Traditionally, insurance has been built on the assumption of independence, and the law of large numbers has governed the determination of premiums. But these days, the increasing complexity of insurance and reinsurance products has led to increased actuarial interest in the modeling of dependent risks. In $[3,4]$, the author considred the problem of optimal allocation of policy limits and deductibles among $n$ random losses $X_{1}, X_{2}, \ldots, X_{n}$ from the viewpoint of a policyholder. The paper concludes that for a risk-averse policyholder, if the sum of policy limits or the sum of deductibles is fixed, then $X_{i} \leq_{s t} X_{j}$ implies that $l_{i}^{\star} \leq l_{j}^{\star}$ and $d_{i}^{\star} \geq d_{j}^{\star}$ when $\left(X_{1}, \ldots, X_{n}\right)$ is comonotonic, wher $l_{i}^{\star}$ and $d_{i}^{\star}$ are the optimal policy limit and the optimal deductible allocated to the $i-t h$ risk.

The losses mentioned above are actually due to both severity and frequency impacts. The policy limit and deductible of a certain risk are for the cumulative losses of the risk during the policy

Received: 30 Oct 2021.

Key words and phrases. comonotonicity; stochastic orders; loss frequency. 
term. In order to study the problem of the optimal allocation of policy limits and deductibles in this situation, an approach to separate the effects of severity and frequency becomes necessary.

In this work, we study the problems of optimal allocation of policy limits and deductibles from the viewpoint of a policyholder, where the dependance structure of losses is unknown for general model. In addition, by applying the bivariate characterizations of stochastic ordering relations, we reconsider the general model and derive some new refined results on ordering of optimal allocations of policy limits and deductibles from the viewpoint of a policyholder.

\section{Preliminary and models}

Suppose that a policyholder is exposed to $n$ random losses, through paying a premium, he could obtain coverage from an insurer. Policy limit and deductible are two common forms of coverage, when the total policy limit or the total deductible is granted, the policyholder can allocate them arbitrarily among the $n$ losses. Let $J$ be an arbitrarily discete index set, representing all random envitronments, and let $\chi=\left\{X_{j}: j \in J\right\}$ be a group of fundamental risk. we assume that $n$ policies of the $n$ losses are effected by the insurance environments. Two states, $J=\{1,2\}$ for insurance, reflect some sort of extra claim generation phenomena (like the influence of weather on car accidents, say). In automobile insurance,

1- might be the risk under normal conditions;

2- might be the risk under bad conditions (slippery roads, foggy days, high traffic volume, and so on). On each policy, the loss under a fixed environment $j \in J$ is characterized by a random variable $X_{j}$. So the loss on each policy is a mixture of some funtamental random variables, i,e., associated with each policy, there exists a random variable $M$ taking values in $J$ such that the loss could be expressed as $X_{M}=\sum_{j \in J} X_{j} \mathbf{1}_{\{M=j\}}$. Recently, effects of financial risks as well as insurance risks have been studied with the scalar product of two random vectors

$$
S_{n}=X_{1} f\left(Y_{1}\right)+X_{2} f\left(Y_{2}\right)+\ldots+X_{n} f\left(Y_{n}\right)
$$

Where: $Y_{i}=\delta_{i} T_{i}, S_{n}$ is a total discounted loss, $X_{i}$ are loss due to the $i-t h$ risk, $T_{i}$ are time of occurrence of $i$-th insured risk and $\delta_{i}$ are discount rate capture the impact of financial environment $\left(X_{i}, T_{i}\right.$ are independent non-negative random variables and $\delta_{i}$ are non-random numbers).

A very good property of the model (M1) (see [6]) is that $X_{i}$ 's characterize the scales of the losses while $f\left(Y_{i}\right)$ characterize the chances of the losses. Also, we will make the following assumptions

$$
\begin{aligned}
& \text { 1.f }\left(Y_{i}\right) \geq 0, \forall Y_{i} \geq 0 \text {, and } \lim _{Y_{i} \rightarrow \infty} f\left(Y_{i}\right)=0 \\
& \text { 2.f }\left(Y_{i}\right) \text { is dereasing and convex function. } \\
& \text { 3. } Y_{1}, Y_{2}, \ldots, Y_{n} \text { are mutually independent. } \\
& \text { 4.A policyholder exposed to risks } X_{1}, X_{2}, \ldots, X_{n} \text { is granted a total of } / \text { dollars }(I \geq 0) \\
& \text { as the policy limit with which he can allocate arbitrarily among the } n \text { risks. }
\end{aligned}
$$


In this situation, if some risk occurs, the insurer will make the payment right after the event of the loss and the insurance coverage for this risk will terminate. However the insurance coverage for the other risks is still in effect. If $\left(I_{1}, \ldots, I_{n}\right)$ are the allocated policy we have $\forall i: I_{i} \geq 0$ and $\sum_{i=1}^{n} l_{i}=l$. When $l$ is $n$-tuple admissible and $\mathcal{S}_{n}(I)$ denote the class of all such $n$-tuples. If $\mathbf{I}=\left(I_{1}, \ldots, I_{n}\right) \in \mathcal{S}_{n}(I)$ is chosen, then the discounted value of benefits obtained from the insurer would be

$$
\sum_{i=1}^{n}\left(X_{M_{i}} \wedge l_{i}\right) f\left(Y_{i}\right)
$$

and hence, the retained discounted loss is

$$
\sum_{i=1}^{n}\left[X_{M_{i}}-\left(X_{M_{i}} \wedge l_{i}\right)\right] f\left(Y_{i}\right)=\sum_{i=1}^{n}\left(X_{M_{i}}-l_{i}\right)_{+} f\left(Y_{i}\right)
$$

Assume that the policyholder is risk-averse, if we take the expected utility of wealth as the optimization criterion for the allocation, then the optimal allocation problem of the policy limits is Problem $\mathcal{L}$ :

$$
\max _{I \in \mathcal{S}_{n}(I)} \mathbb{E}\left[u\left(w-\sum_{i=1}^{n}\left(X_{M_{i}}-l_{i}\right)_{+} f\left(Y_{i}\right)\right)\right]
$$

where $u($.$) is the utility function of the policyholder (u($.$) is increasing and concave) and w$ is the wealth (after the premium). On the other hand, instead of policy limits, we assume that $d$ $d=\left(d_{1}, \ldots, d_{n}\right) \in S_{n}(d)$ are the allocated deductibles, $d_{i} \geq 0$ for all $i, d_{1}+\ldots+d_{n}=d$. And the discounted benefits obtained from the insurer would be

$$
\sum_{i=1}^{n}\left(X_{M_{i}}-d_{i}\right)_{+} f\left(Y_{i}\right)
$$

hence, the optimal allocation problem of the policy deductibles is Problem $D$ :

$$
\max _{d \in \mathcal{S}_{n}(d)} \mathbb{E}\left[u\left(w-\sum_{i=1}^{n}\left(X_{M_{i}} \wedge d_{i}\right) f\left(Y_{i}\right)\right)\right]
$$

where $u($.$) and w$ admit the same interpretations as that in Problem $\mathcal{L}$. Inheriting from the dependence structure in $\chi=\left\{X_{j}: j \in J\right\}$, the losses $\left\{X_{M_{i}}, i=1, \ldots, n\right\}$ also have an unknown dependence structure. Like work [2], we will take a conservative attitude towards this uncertainty, and view the most unfavorable situation as the actual dependence structure. Therefore, Problem $\mathcal{L}$ is modified to:

$$
\max _{I \in \mathcal{S}_{n}(I)} \min _{\left(X_{M_{1}}, X_{M_{2}}, \ldots, X_{M_{n}}\right)} \mathbb{E}\left[u\left(w-\sum_{i=1}^{n}\left(X_{M_{i}}-l_{i}\right)_{+} f\left(Y_{i}\right)\right)\right]
$$

and Problem $D$ is modified to:

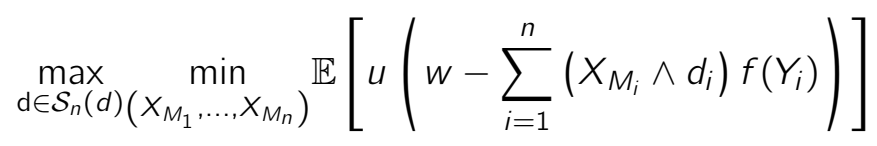


In order to make our discussion proceed, we firstly solve the "min" parts of the problems mentioned above, i.e., to identify the worst dependence structure that minimizes the expected utility. Note that $u($.$) and w$ are the utility function (increasing and concave) and the wealth (after premium) respectively, then $\widetilde{u}(x): x \rightarrow-u(w-x)$ is an increasing convex function. So the two problems are equivalent to

Problem $\mathcal{L}^{\prime}$ :

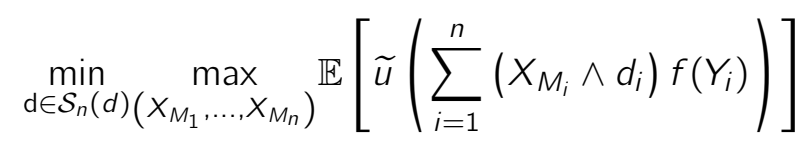

Now, the "min" parts are transformed into "max" parts. The following two propositions are provided for solving the "max" parts firstly.

Proposition 3.1. Suppose thate $\widetilde{u}$ is increasing and convex. Let $\left\{X_{j}^{c}, j \in J\right\}$ be an any comonotonic copy of $\left\{X_{j} ; j \in J\right\}$,i,e., they have the same marginal distributions, and $\left\{X_{j}^{c} ; j \in J\right\}$ is comonotonic and jointly measurable. Assume that both $\left\{X_{j} ; j \in J\right\}$ and $\left\{X_{j}^{c} ; j \in J\right\}$ are independent of $\left\{M_{1}, \ldots, M_{n}\right\}$ and $\left\{Y_{1}, \ldots, Y_{n}\right\}$. Then

$$
\mathbb{E}\left[\widetilde{u}\left(\sum_{i=1}^{n}\left(X_{M_{i}}-l_{i}\right)_{+} f\left(Y_{i}\right)\right)\right] \leq \mathbb{E}\left[\widetilde{u}\left(\sum_{i=1}^{n}\left(X_{M_{i}}^{c}-l_{i}\right)_{+} f\left(Y_{i}\right)\right)\right]
$$

Proof. Fix any $j_{1}, \ldots, j_{n} \in J$, and $t_{1}, \ldots, t_{n} \geq 0$. According to the hypothesis, the collection $\left\{\left(X_{j_{1}}^{c}-I_{1}\right)_{+} f\left(Y_{1}\right), \ldots,\left(X_{j_{n}}^{c}-I_{n}\right)_{+} f\left(Y_{n}\right)\right\}$ is also comonotonic. Therefore,

$$
\sum_{i=1}^{n}\left(X_{j_{i}}-l_{i}\right)_{+} f\left(Y_{i}\right) \leq_{c x} \sum_{i=1}^{n}\left(X_{j_{i}}^{c}-l_{i}\right)_{+} f\left(Y_{i}\right)
$$

which follows from Lemma 2.2. Sincee $\widetilde{u}($.$) is increasing and convex, then$

$$
\mathbb{E}\left[\widetilde{u}\left(\sum_{i=1}^{n}\left(X_{j_{i}}-l_{i}\right)_{+} f\left(Y_{i}\right)\right)\right] \leq_{c x} \mathbb{E}\left[\widetilde{u}\left(\sum_{i=1}^{n}\left(X_{j_{i}}^{c}-l_{i}\right)_{+} f\left(Y_{i}\right)\right)\right]
$$

Hence, in view of the fact that $\left\{M_{1}, \ldots, M_{n}\right\}$ and $\left\{Y_{1}, \ldots, Y_{n}\right\}$ are independent, and they are independent of $\left\{X_{j} ; j \in J\right\}$, we

have

$$
\begin{aligned}
\mathbb{E}\left[\widetilde{u}\left(\sum_{i=1}^{n}\left(X_{M_{i}}-l_{i}\right)_{+} f\left(Y_{i}\right)\right)\right] & =\mathbb{E}\left\{\mathbb{E}\left[\widetilde{u}\left(\sum_{i=1}^{n}\left(X_{M_{i}}-l_{i}\right)_{+} f\left(Y_{i}\right)\right) \mid M_{1}, \ldots, M_{n}, Y_{1}, \ldots, Y_{n}\right]\right\} \\
& \leq \mathbb{E}\left\{\mathbb{E}\left[\widetilde{u}\left(\sum_{i=1}^{n}\left(X_{M_{i}}^{c}-l_{i}\right)_{+} f\left(Y_{i}\right)\right) \mid M_{1}, \ldots, M_{n}, Y_{1}, \ldots, Y_{n}\right]\right\} \\
& =\mathbb{E}\left[\widetilde{u}\left(\sum_{i=1}^{n}\left(X_{M_{i}}^{c}-l_{i}\right)_{+} f\left(Y_{i}\right)\right)\right] .
\end{aligned}
$$

Proposition 3.2. Suppose that $\widetilde{u}$ is increasing and convex. Let $\left\{X_{j}^{c} ; j \in J\right\}$ be a comonotonic copy of $\left\{X_{j} ; j \in J\right\}$, assume that both $\left\{X_{j} ; j \in J\right\}$ and $\left\{X_{j}^{c} ; j \in J\right\}$ are independent of 
$\left\{M_{1}, \ldots, M_{n}\right\}$ and $\left\{Y_{1}, \ldots, Y_{n}\right\}$. Then,

$$
\mathbb{E}\left[\widetilde{u}\left(\sum_{i=1}^{n}\left(X_{M_{i}} \wedge d_{i}\right) f\left(Y_{i}\right)\right)\right] \leq \mathbb{E}\left[\widetilde{u}\left(\sum_{i=1}^{n}\left(X_{M_{i}}^{c} \wedge d_{i}\right) f\left(Y_{i}\right)\right)\right]
$$

Remark 3.2. Owing to these two propositions, we will assume that $\left\{X_{j} ; j \in J\right\}$ is comonotonic from now on. One important

consequence is that if $j_{1} \leq J_{2}$, then $X_{j_{1}} \leq_{s t} X_{J_{2}}$, and hence, $X_{j_{1}} \leq X_{J_{2}}$ on $\Omega$ by comonotonicity, which means that every

realization of $\left\{X_{j} ; j \in J\right\}$ is an increasing real-valued function on $J$. Actually, following from the former three conditions listed in the beginning of this section, the existence of the comonotonic and jointly measurable copy $\left\{X_{j}^{c} ; j \in J\right\}$ can be proved, and the proof also appears in [4].

\section{REFERENCES}

[1] F. Hua, R. Wang, Optimal allocation of policy limits and deductibles in a model with mixture risks and discount factors, J. Comput. Appl. Math. 234 (2010) 2953-2961 https://doi.org/10.1016/j . cam. 2010.04.007.

[2] K.C. Cheung, Optimal portfolio problem with unknown dependency structure, Insurance: Math. Econ. 38 (1) (2006) 167-175 https://doi.org/10.1016/j.insmatheco.2005.08.006.

[3] K.C. Cheung, Optimal allocation of policy limits and deductibles, Insurance: Math. Econ. 41 (3) (2007) 382 391 https: //doi.org/10.1016/j.insmatheco.2006.11.010.

[4] K.C. Cheung, H.L. Yang, Ordering of optimal portfolio allocation in a model with mixture of fundamental risks, J. Appl. Probab. 45 (1) (2008) 55-66 https://doi.org/10.1017/S0021900200003958.

[5] L. Hua, K.C. Cheung, Stochastic orders of scalar products with applications, Insurance: Math. Econ. 42 (3) (2008) 865-872 https://doi.org/10.1016/j.insmatheco.2007.10.004.

[6] M. Bouhadjar, H. Zeghdoudi, M. R. Remita ,On Stochastic Orders and their Applications: Policy Limits and Deductibles. Appl. Math. Inf. Sci. 10 (4) (2016) 1385-1392 https://doi.org/10.18576/amis/100417. 